\title{
La rehabilitación cardiorrespiratoria tras un ictus: hacia un nuevo paradigma
}

\section{Cardiorespiratory rehabilitation after a stroke: towards a new paradigm}

\author{
A.Lista-Paz ${ }^{\mathrm{a}, \mathrm{b}}$ \\ ${ }^{a}$ Departamento de Fisioterapia, Medicina y Ciencias Biomédicas, Facultad de Fisioterapia de la Universidade da \\ Coruña, A Coruña, España \\ ${ }^{b}$ Grupo de investigación en Intervención Psicosocial y Rehabilitación Funcional
}

El ictus o accidente cerebrovascular (ACV) se engloba dentro de las enfermedades cardiovasculares, definidas por la Organización Mundial de la Salud como aquellas que afectan al corazón y a los vasos sanguíneos. Dentro de este grupo, las enfermedades cerebrovasculares (ECV) constituyen un problema sociosanitario de primer orden en todo el mundo, siendo la primera causa de discapacidad y dependencia en el adulto a nivel mundial. Según datos publicados por la Organización Mundial de la Salud, las ECV son la segunda causa de muerte (tras la cardiopatía isquémica) en todos los países, especialmente en aquellos con niveles de ingreso medio y alto ${ }^{1}$. Datos publicados por la Asociación Americana del Corazón señalan que cada 40 segundos alguien sufre un ictus en Estados Unidos. En España, según el estudio Iberictus ${ }^{2}$, la tasa cruda de incidencia del ACV es de 187 casos nuevos por cada 100.000 habitantes.

Para conocer en profundidad la medida real del impacto del ACV, es necesario señalar el alto riesgo de recidiva al que están expuestos los pacientes que ya han sufrido un primer episodio cerebrovascular. Los resultados de un metaanálisis realizado en el año $2011^{3}$ muestran que el riesgo de sufrir un segundo ACV tras el primer ictus es del 3,1\% (IC95\% = 1,7-4,4) a los 30 días, del 11,1\% (IC95\% = 9,0-13,3) al año, aumentando a un 26\% (IC95\% $=20,1-32,8)$ a los 5 años y a un $40 \%($ IC95\% $=27,2-51,2)$ a los 10 años.

El estudio de los datos epidemiológicos del ACV pone de manifiesto la necesidad de enfatizar la implementación de programas, no solo de prevención primaria sino también de prevención secundaria, una vez instaurada la ECV, ya que el elevado riesgo de recidiva del ACV tiene un origen claramente multifactorial, asociándose a un mayor número de factores de riesgo cardiovascular. El $80 \%$ de los factores de riesgo cardiovascular son modificables, quedando relegados al $20 \%$ restante factores como la edad, el sexo y la genética. Entre los factores de riesgo cardiovascular modificables cabe destacar el sedentarismo o inactividad física, ya que ha demostrado tener una relación inversamente proporcional con el ACV: niveles de actividad altos o moderados se asocian con menor riesgo de ictus y niveles de actividad física insuficientes condicionan un mayor riesgo de ACV. Además, el sedentarismo guarda una correlación directa con otros factores de riesgo cardiovasculares como la hipertensión arterial, la diabetes mellitus, la dislipemia y la obesidad, que en la mayor parte de los casos continúan presentes en las 
personas que han sufrido un ictus. Es por ello que el ejercicio terapéutico se erige como piedra angular tanto en la prevención primaria como en la prevención secundaria de las ECV ${ }^{4}$.

Para comprender en mayor profundidad la importancia de los programas de prevención secundaria en los pacientes con ECV es fundamental atender no solo a sus citados factores de riesgo cardiovascular, sino también a las consecuencias tras un ACV. Más del $90 \%$ de los pacientes que sufren un ACV presentan algún tipo de discapacidad. Las diversas alteraciones funcionales que se producen tras sufrir un $\mathrm{ACV}$, son abordadas desde diferentes corrientes terapéuticas. En un sentido más amplio e integral, cada vez más, se aboga por la neurorrehabilitación, desde el equipo multi-, inter- y transdisciplinar, en donde el paciente y su entorno familiar, constituyen el centro del equipo de salud. Históricamente y todavía hoy, son muchos y diversos los métodos y técnicas en rehabilitación neurológica que se centran en el abordaje de las disfunciones sensitivo-motoras, persiguiendo la recuperación de la funcionalidad del paciente, facilitando así su reintegración en la vida social y mejorando su calidad de vida. Sin embargo, el sistema cardiorrespiratorio ha sido y continúa siendo el gran olvidado en esta población, especialmente una vez instaurada la cronicidad, pese a las implicaciones que una disfunción de la función cardiopulmonar y de la musculatura respiratoria podrían tener a nivel sistémico.

Desde el punto de vista cardiovascular es importante destacar que la fatiga, entendida como la dificultad para iniciar o mantener un esfuerzo, es una de las principales consecuencias autoinformadas por las personas que han sufrido un ictus, especialmente durante la fase crónica. A su vez, la capacidad cardiorrespiratoria, medida en términos de consumo pico de oxígeno $\left(\mathrm{VO}_{2}\right.$ pico $)$, se ve reducida hasta en un $87 \%$ tras un $\mathrm{ACV}^{5}$. Se ha demostrado que un $\mathrm{VO}_{2}$ pico de $20 \mathrm{ml} \cdot \mathrm{kg}^{-1} \cdot \mathrm{min}^{-1}$ es necesario para el desempeño independiente de las actividades de la vida diaria, sin embargo, los rangos alcanzados por personas que han sufrido un ACV se sitúan lejos de dichos valores, lo cual conduce a largos períodos de tiempo inactivos, con el consiguiente daño para el sistema cardiovascular, de por sí afectado por la enfermedad ${ }^{6}$.

El sistema respiratorio es una de las principales vías de obtención de los elementos necesarios para la vida, entre ellos, garantiza el aporte de oxígeno a todos los tejidos corporales. Se ha sugerido que la optimización del funcionamiento del sistema respiratorio, especialmente de la función pulmonar, tras un $\mathrm{ACV}$, podría incrementar los resultados de los programas de rehabilitación, durante los cuales se está produciendo una solicitación constante de diferentes grupos musculares. En relación a esto, se ha documentado la presencia de disnea y fatiga en los supervivientes a un ACV durante el desempeño de tareas que demandan altos esfuerzos, incluso pudiendo limitar en algunos casos sus actividades de la vida diaria, lo cual está directamente relacionado con la baja participación social de estos pacientes y la merma de su calidad de vida ${ }^{7}$.

La evaluación y tratamiento del sistema respiratorio han sido abordados durante las primeras fases del $\mathrm{ACV}$, ya que suponen en muchas ocasiones, un riesgo vital para el paciente durante los primeros días de evolución. Así, son conocidos los patrones respiratorios alterados de la primera fase del ACV como la taquipnea y la respiración de Cheyne-Stokes, el alto riesgo de aspiraciones, los trastornos respiratorios del sueño, la elevada incidencia de infecciones respiratorias, tales como la neumonía, etc. ${ }^{8}$. A su vez, existe acuerdo entre la comunidad científica acerca de la debilidad de la musculatura respiratoria durante la fase aguda y subaguda del ictus y también de su déficit de resistencia a la fatiga, objetivados a través de la determinación de la presión inspiratoria máxima (PIM), presión espiratoria máxima (PEM) y de la máxima ventilación voluntaria ${ }^{9}$.

Sin embargo, cuando se trata de pacientes con ACV en estadio crónico existe mayor controversia sobre las implicaciones del mismo a nivel respiratorio. Las últimas investigaciones apuntan a la existencia de un patrón ventilatorio restrictivo. Además se ha confirmado que existe debilidad de la musculatura respiratoria y baja resistencia a la fatiga, siendo la PIM, la PEM y la máxima ventilación voluntaria significativamente más bajas en estos sujetos que cuando se comparan con personas sanas apareadas por edad y sexo ${ }^{10}$. 
Habiendo analizado las consecuencias que el ACV puede tener sobre el sistema cardiorrespiratorio es fácil entender cómo los programas de rehabilitación cardiorrespiratoria pueden contribuir a mejorar el proceso de recuperación de estos pacientes. Dichos programas se caracterizan por su abordaje multifactorial, vertebrándose en dos ejes fundamentales: la prescripción de ejercicio terapéutico y los programas de educación al paciente (dentro del cual podríamos incluir un programa de deshabituación tabáquica, plan nutricional, medidas de control del estrés y, en términos generales, la identificación y manejo de los factores de riesgo cardiovascular).

Especial mención, por la alta evidencia científica que ha demostrado, merece la prescripción del ejercicio terapéutico en el ACV, desde la fase subaguda a la más crónica, habiéndose registrado numerosos beneficios, tanto desde el punto de vista cardiovascular como en términos de fuerzaresistencia. Antiguamente, tras un ictus, se evitaba la recomendación de ejercicio físico, pues se sostenía la hipótesis de que aumentaba la espasticidad, la rigidez y el dolor, al tiempo que empeoraba el patrón de marcha. Sin embargo, los estudios más recientes han desmentido dichas creencias, ya que la espasticidad solo aumenta durante la realización de la actividad, volviendo a los valores anteriores una vez finalizada la misma, y sin que el dolor se agudice. Entre los beneficios del entrenamiento progresivo de fuerzaresistencia destacan el incremento de la fuerza muscular, el pico de potencia y una mayor activación neuromuscular, siendo las intervenciones con actividades orientadas a la tarea las que han evidenciado mejores resultados. Además, tanto el entrenamiento de fuerza-resistencia como el entrenamiento aeróbico, han demostrado resultados positivos sobre la capacidad de marcha tras un ACV, aumentando tanto la velocidad como la distancia recorrida. Por su parte, los programas de entrenamiento aeróbico mejoran los parámetros cardiorrespiratorios (en términos de $\left.\mathrm{VO}_{2}\right)^{11}$.

Por otra parte, cada vez son más los autores que tratan de estudiar la efectividad del entrenamiento de la musculatura respiratoria en pacientes con ACV y sus repercusiones sobre parámetros funcionales. Un metaanálisis publicado en 2016 realizado a partir de siete ensayos clínicos controlados y aleatorizados concluye que el entrenamiento de la musculatura respiratoria tras un ACV mejora significativamente la PIM, incrementa la capacidad vital forzada y el volumen espiratorio máximo en el primer segundo, así como la tolerancia al ejercicio $^{12}$. A su vez, una revisión sistemática publicada en el mismo año concluye que el entrenamiento de la musculatura respiratoria supone un incremento significativo tanto de la PIM como de la PEM, al tiempo que disminuye las complicaciones respiratorias ${ }^{13}$. Sin embargo, es importante destacar que en ambos trabajos se han incluido investigaciones realizadas sobre pacientes en estadio agudo, subagudo y crónico, por lo que se trata de una población heterogénea, difícilmente comparable en cuanto a las presiones respiratorias máximas que mostraron de base $\mathrm{e}^{12,13}$. Además, existen diferencias sustanciales en los protocolos de entrenamiento de la musculatura respiratoria, oscilando la carga programada entre el $30 \%$ y el $60 \%$ de la PIM, con programas que duran desde 3 a 8 semanas. Asimismo, en el caso de Menezes et al. ${ }^{13}$, sería importante profundizar en la relevancia clínica de los datos aportados, pues se informa sobre un incremento medio de la PIM de $7 \mathrm{cmH}_{2} \mathrm{O}$ y de $13 \mathrm{cmH}_{2} \mathrm{O}$ en el caso de la PEM. Por tanto, si bien parece que el entrenamiento de la musculatura respiratoria reporta beneficios en las diferentes fases de recuperación del ictus, se requieren más estudios primarios que arrojen luz sobre los parámetros de entrenamiento más apropiados en pacientes con ACV.

En conclusión, la evaluación pormenorizada del sistema cardiorrespiratorio es necesaria en personas que han sufrido un ictus. Además, la implementación de programas de rehabilitación cardiorrespiratoria tras un ACV se plantea como una opción terapéutica basada en la evidencia para mejorar la condición cardiorrespiratoria de los pacientes, permitiéndoles una vida más activa, con el consiguiente incremento de su participación social y por ende, de su calidad de vida. Asimismo, y siguiendo el modelo de la rehabilitación pulmonar, se ha justificado la importancia de estudiar los efectos que el entrenamiento de la musculatura respiratoria pueda tener cuando se integra dentro de un programa de ejercicio terapéutico que englobe entrenamiento de fuerza-resistencia de la musculatura periférica y trabajo aeróbico. 


\section{Bibliografía}

1. Mendis S, Puska P, Norrving B. Global Atlas on Cardiovascular Disease Prevention and Control [Internet]. Geneva: World Health Organization; 2011 [citado 19 Junio 2018]. Disponible en: file:///C:/Users/Fisio2/Downloads/9789241564373_eng.pdf

2. J. Díaz, J. Egido, R. Gabriel, G. Barberá, B. Fuentes, C. Fernández. Stroke and transient ischemic attack incidence rate in Spain: the IBERICTUS study. Cerebrovasc Dis., 34 (2012), pp. 272-281

3. K.M. Mohan, C.D.A. Wolfe, A.G. Rudd, P.U. Heuschmann, P.L. Kolominsky-Rabas, A.P. Grieve. Risk and cumulative risk of stroke recurrence: a systematic review and meta-analysis. Stroke., 42 (2011), pp. 1489-1494

4. W.N. Kernan, B. Ovbiagele, H.R. Black, D.M. Bravata, M.I. Chimowitz, M.D. Ezekowitz, et al. Guidelines for the prevention of stroke in patients with stroke and transient ischemic attack: a guideline for healthcare professionals from the American Heart Association/American Stroke Association. Stroke., 45 (2014), pp. 2160-2236

I. Baert, D. Daly, E. Dejaeger, C. Vanroy, Y. Vanlandewijck, H. Feys. Evolution of cardiorespiratory fitness after stroke: a 1-year follow-up study. Influence of prestroke patients' characteristics and stroke-related factors. Arch Phys Med Rehabil., 93 (2012), pp. 669-676

5. F.M. Ivey, R.F. Macko, A.S. Ryan, C.E. Hafer Macko. Cardiovascular health and fitness after stroke. Top Stroke Rehabil., 12 (2005), pp. 1-16

6. B. Lanini, F. Gigliotti, C. Coli, R. Bianchi, A. Pizzi, I. Romagnoli, et al. Dissociation between respiratory effort and dyspnoea in a subset of patients with stroke. Clin Sci., 103 (2002), pp. 467-473

7. Y. Hannawi, B. Hannawi, C.P.V. Rao, J. Suarez, E. Bershad. Stroke-associated pneumonia: major advances and obstacles. Cerebrovasc Dis., 35 (2013), pp. 430-443

8. B. Lanini, R. Bianchi, I. Romagnoli, C. Coli, B. Binazzi, F. Gigliotti, et al. Chest wall kinematics in patients with hemiplegia. Am J Respir Crit Care Med., 168 (2003), pp. 109-113

A. Lista, L. González, S. Ortigueira, J. Saleta, C. Moreno. Respiratory muscle strength in chronic stroke survivors and its relation with the 6-minute walk test. Arch Phys Med Rehabil., 97 (2016), pp. 266-272

9. S. Billinger, R. Arena, J. Bernhardt, J. Eng, B. Franklin, C. Johnson, et al. Physical activity and exercise recommendations for stroke survivors: a statement for healthcare professionals from the American Heart Association/American Stroke Association. Stroke., 45 (2014), pp. 25322553

10. M. Gomes, M. Saquetto, C. Silva, V. Carvalho, N. Ribeiro, C. Conceição. Effects of respiratory muscle training on respiratory function. Respiratory muscle strength, and exercise tolerance in patients poststroke: A systematic review with meta-analysis. Arch Phys Med Rehabil., 97 (2016), pp. 1994-2001

11. K. Menezes, L. Nascimento, L. Ada, J. Polese, P. Avelino, L. Teixeira Salmela. Respiratory muscle training increases respiratory muscle strength and reduces respiratory complications after stroke: a systematic review. J Physiother., 62 (2016), pp. 138-144 